\title{
ОПЕРАТИВНОЕ И ДОЛГОСРОЧНОЕ ПРОГНОЗИРОВАНИЕ ПРОДУКТИВНОСТИ ПОСЕВОВ НА ОСНОВЕ МАССОВЫХ РАСЧЕТОВ ИМИТАЦИОННОЙ МОДЕЛИ АГРОЭКОСИСТЕМЫ В ГЕОИНФОРМАЦИОННОЙ СРЕДЕ \\ (обзор)
}

\author{
В.П. ЯКУШЕВ, В.В. ЯКУШЕВ, В.Л. БАДЕНКО, Д.А. МАТВЕЕНКО, \\ Ю.В. ЧЕСНОКОВ
}

Системы земледелия, представляющие собой комплекс взаимосвязанных агротехнических, мелиоративных и организационных мероприятий, обеспечивающих эффективное использование агроландшафтов, сохранение и повышение плодородия почвы, а также получение высоких урожаев, рассматриваются как инструмент управления сельскохозяйственным производством, особенно актуальный в современных изменяющихся социально-экономических и природно-климатических условиях. Оптимизация таких систем в значительной степени строится на расчетах числовых значений параметров агроэкосистем и прогнозировании их динамики с применением математических моделей. В растениеводстве получили развитие статистические и динамические имитационные прогнозные модели. Последние модели более точные, адаптивные и позволяют описать развитие агроэкосистем при нестабильных климатических условиях и под воздействием различных агротехнических мероприятий. Математические модели широко обсуждаются в научной литературе по экологии, почвоведению, растениеводству. Основные проблемы регионального планирования систем земледелия на средне- и долгосрочную перспективу могут быть решены на основе моделирования в среде геоинформационных систем. Обзор подходов к прогнозированию продуктивности посевов на основе массовых расчетов по имитационной модели агроэкосистемы в геоинформационной среде позволяет определить, как можно их использовать для обоснования систем земледелия. При этом по пространственному охвату методы моделирования делятся на макромасштабные, мезомасштабные и микромасштабные. В общем случае для разных масштабов используются неодинаковые подходы. Для создания адекватной этим подходам универсальной среды массовых расчетов по динамическим моделям агроэкосистем для разных уровней пространственного охвата предлагается использовать соответствующие компьютерные оболочки для поливариантных расчетов - универсальную среду моделирования, для которой представлены требования для проведения расчетов по разным моделям от различных поставщиков. Решение проблемы формирования набора точек для расчета по модели состоит в том, что они должны находиться на возделываемых сельхозугодиях и адекватно представлять почвенные и климатические условия региона.

Ключевые слова: агроэкосистемы, имитационное моделирование, массовые вычисления, прогнозирование, геоинормационные системы, системы земледелия.

Взгляд на роль и место динамических имитационных моделей продукционного процесса растений в практической агрономии и теоретической агроэкологии в последнее время существенно изменился (1-3). Сфера применения таких моделей, которая традиционно ограничивалась научными исследованиями агроэкосистем, все более расширяется в направлении практического применения в реальных системах принятия решений (4). Следствием повышения качества моделей агроэкосистем, а также развития подходов в теории математического моделирования процессов и явлений в системе «почва-растение-атмосфера», является то, что перечень сельскохозяйственных культур и почвенно-климатических условий, используемых в исследованиях с использованием семейств универсальных экофизиологических моделей, становится практически неограниченным (5). Кроме того, большинство современных моделей агроэкосистем основано на все бо́льшем числе определяющих процессов и явлений физической и биологической природы (4-7). Поэтому такие модели, наряду с продуктивностью

* Выполнено за счет федерального бюджета по соглашению о предоставлении субсидии от 10 декабря 2019 года № 05.607.21.0302, уникальный идентификатор проекта RFMEFI60719X0302. 
(урожайностью), позволяют количественно оценивать и иные характеристики агроэкосистем. В модельных прогнозах происходит переход от единичных сезонов вегетации к многолетним непрерывным расчетам севооборотов с учетом плодосмены культур (4). Также все более популярна идея существенного варьирования временно́го и пространственного масштаба динамических моделей, в частности выполнения расчетов не только для одной культуры в одной географической точке для одного срока вегетации, но и для произвольно выбранных периодов времени и территорий. Иначе говоря, под пространственным расширением понимается одновременный расчет пространственно-одномерной модели не только для одной выбранной представительной точки земной поверхности с конкретно заданными свойствами почвы и местности, но и для представительного набора таких точек, формирующих неоднородный агроландшафт $(6,7)$. При этом в отличие от классических одномерных моделей однородного сельскохозяйственного посева, динамические модели агроэкосистем третьего поколения позволяют описывать адаптивно-ландшафтные системы земледелия (5). Это стало возможно благодаря существенному прогрессу в вычислительных технологиях, практически снявших ограничения на эффективность и быстродействие численных алгоритмов, заложенных в модель.

Далее нами представлен анализ общесистемных методических подходов, основанных, в том числе на опыте авторов обзора. Рассмотрены перспективы разработки и использования имитационных динамических моделей агроэкосистем и специализированных средств автоматизации вычислительного эксперимента с этими моделями для решения различных задач оперативного и долгосрочного регионального прогнозирования продуктивности посевов разного временно́го и пространственного масштаба.

Динамические модели агроэкосистем в системах приня тия решений. Использование моделирования в системах принятия решений требует адекватного информационного обеспечения, для чего повсеместно в практику растениеводства и полевых опытов внедряются все новые инструменты оперативного мониторинга состояния и автоматизации измерений, включая бесконтактные методы, дистанционное зондирование и портативные автоматические метеостанции (7-11). Такие инструментальные методы повышают качество информационного обеспечения моделирования продукционного процесса растений, а также достоверность данных, используемых для параметрической идентификации параметров и верификации алгоритмов в моделях агроэкосистем посредством ассимиляции пространственно-распределенных данных измерений. При этом традиционные статистически обоснованные регрессионные модели агроэкосистем разной сложности в настоящий момент представляют собой самый распространенный инструмент оценки урожайности культур на больших площадях в задачах регионального средне- и долгосрочного планирования (12-16). Такие модели до сих пор служат основой нормативных методик, согласно которым проводится оценка потенциальной продуктивности главных хозяйственных культур для текущего сезона вегетации по регионам. Однако в таких моделях влияние многих факторов (физических, климатических и технологических) могут учитываться не в полной мере. Поэтому динамические имитационные модели агроэкосистем выступают перспективным инструментом прогнозирования, позволяющим адекватно ответить на многочисленные вызовы (17-20). Методология использования таких динамических моделей агроэкосистем для задач регионального планирования в настоящее время практически отсутствует, а примеры успешного средне- и долгосрочного прогнозирования малочисленны, что во многом объясняется 
тем, что адекватное пространственно-временно́е масштабирование требует адаптации и модификации внутренней логики существующих моделей и инфраструктуры компьютерных экспериментов (21-23). Такая модификация связана с необходимостью адекватного моделирования последовательности культур в многолетнем севообороте и учета разнообразия долговременных агротехнологических мероприятий (24-27). Решением может служить использование одной модели продукционного процесса агроэкосистем для всего разнообразия возделываемых культур с общей структурой данных и стандартной архитектурой, что позволит использовать ее для планирования региональных систем земледелия.

В лаборатории математического моделирования агроэкосистем Агрофизического НИИ (г. Санкт-Петербург) в течение 40 лет успешно ведутся работы по созданию и усовершенствованию семейства моделей продукционного процесса AGROTOOL $(28,29)$. К настоящему времени разработана версия компьютерной модели третьего уровня продуктивности Agrotool v3.5 по классификации C.T. de Wit (30).

В общем виде для расчетного обоснования севооборотов в системах земледелия на основе анализа рассмотренных выше публикаций (1-30) можно сформулировать следующие требования к моделям агроэкосистем. Модель должна учитывать воздействие культуры-предшественника, включая пожнивные остатки, симбиотическую азотфиксация бобовыми растениями, изменение агрофизических и агрохимических свойств почвы и т.д.; включать описание абиотических процессов вне периода вегетации («перезимовка»), такие как нарастание и стаивание снежного покрова, промерзание и прогрев почвы и т.п.; учитывать возможное явное указание доз и сроков агротехнических мероприятий, реактивные режимы управления, такие как автоматический полив, назначение даты сева и т.д.; быть устойчивой к отсутствию фактической информации. Упомянутое семейство моделей AGROTOOL и версия Agrotool v3.5 полностью соответствуют этим требованиям.

Также жесткие требования для расширения сферы применения моделей агроэкосистем должны быть предъявлены к среде исполнения моделей - так называемым компьютерным оболочкам $(31,32)$. Принципиальное требование связано с необходимостью обеспечения поливариантного расчета модели в автоматическом режиме для большого количества альтернативных вариантов с подготовленными заранее наборами входных данных, а также с возможностью последовательного или параллельного запуска расчетов модели. Необходимостью становится также наличие в среде исполнения моделей удобной связи с геоинформационными системами (ГИС) для пространственной привязки данных о почвенных разностях и цифровой модели рельефа, а также визуализации результатов моделирования в виде тематических карт оцениваемых показателей (33). Кроме того, следует учитывать, что большинство задач прогнозирования требует наличия процедур автоматического формирования «синтетических» входных данных модели (например, генератора погодных характеристик), обладающих пространственной и временной связностью $(34,35)$.

Агрономические динамические модели продуктивности традиционно рассматриваются как одномерные, или одноточечные (5). Иными словами, каждый конкретный вариант запуска модели требует в качестве входных данных сведений о конкретном участке территории, для которой производится расчет (характеристики почвы, рельеф, погода и т.д.). Распространение сферы приложения модели на массовые пространственные расчеты для географически распределенных территорий требует сопряжения 
динамических моделей агроэкосистемы с ГИС. Действительно, подобное объединение позволяет изучать агроэкосистемы одновременно во временно́й и пространственной развертке $(33,36,37)$. Практическая направленность и востребованность подобных исследований очевидна. Так, возможность прогнозировать потери сельскохозяйственного производства, связанные с изменениями внешних условий, в первую очередь природных, посредством подбора систем земледелия позволяет принимать превентивные меры для поддержания некоторого уровня продовольственной безопасности $(37,38)$. В регионах рискованного земледелия системы раннего предупреждения, основанные на дистанционном зондировании, могут помочь заблаговременно выявить возникающую проблему $(39,40)$. Зачастую подобные системы основаны на мониторинге погодных и сельскохозяйственных условий в течение вегетационного периода и включают в себя регионально откалиброванные модели культур для оценки неопределенности урожайности (41). При этом прогнозы урожайности можно сделать до посадки или в течение текущего вегетационного периода, а результаты, полученные с помощью моделей, использовать для принятия управленческих решений и оперативного предоставления альтернативных вариантов ведения хозяйства (42).

Но пространственная масштабируемость модели неизбежно приводит к тому, что при расширении сферы использования от точки до территории резко возрастает требуемый объем информационной поддержки и вычислительных ресурсов (43). Более того, оформились принципиально новые направления потенциального приложения соответствующих технологий в растениеводстве и производстве сельскохозяйственной продукции например, использование данных дистанционного зондирования посевов $(14,39,41,44,45)$, в том числе для точного земледелия (10). В определенной степени острота проблемы получения всей необходимой информации для массовых пространственных расчетов снята в задаче оценки влияния потенциальных климатических изменений на продуктивность и устойчивость агроландшафта $(46,47)$. Для конкретного варианта расчета необходимый набор входных метеорологических данных (представительная выборка сценариев погодных реализаций климата будущего) для произвольной пространственной сетки опорных точек можно получить из базы данных IPCC (Intergovernmental Panel on Climate Change, https://www.ipcc.ch/). Однако до сих пор не закончена разработка методики и не создан адекватный инструментарий для оперативного модельного прогнозирования продуктивности сельскохозяйственных культур при разном пространственном охвате территорий. Проблема масштабируемости и универсализации соответствующих программных и методических решений приобретает в последнее время все более критическую остроту $(48,49)$. Достаточно сказать, что в рамках проекта MACSUR (Modeling European Agriculture with Climate Change for Food Security, https://www.macsur.eu/) - общеевропейского информационного портала об использовании имитационных моделей для информационной поддержки процессов адаптации сельского хозяйства Европы к последствиям антропогенных климатических изменений выделена специальная рабочая группа Scale-It! (http://www.scale-it.net/). Ее задача координировать европейские исследования по использованию моделей продуктивности для решения практических задач поддержки решений в различном пространственном масштабе (50). Сфера деятельности этой группы включает организацию проектов ансамблевых расчетов, агрегацию специфических входных данных с разных географических локаций, научное обоснование методов пространственной дискретизации и пространственной интерполяции входных данных для моделей с оценкой степени неопределенности 
получаемых результатов, сбор информации о персоналиях, моделях, подходах, предоставление оперативной информации о вызовах и достижениях в обсуждаемой области исследований.

Среди наиболее значимых прикладных результатов европейских исследований по динамическим моделям продуктивности для комплексного описания агроэкосистем в региональном масштабе следует отметить среду LandCare-DSS (http://www.landcare2020.de/), разработанную в институте системного моделирования ландшафтов Центра агроландшафтных исследований в г. Мюнхеберге (Leibniz Centre for Agricultural Landscape Research ZALF, Германия) (51). В настоящее время это, пожалуй, единственная подобная разработка, доведенная до уровня конечного программного продукта с приемлемой степенью автоматизации (52). К уникальным характеристикам LandCare-DSS следует отнести встроенный собственный ГИС-интерфейс, интеграцию с экономическими моделями, а также использование в рамках единой архитектуры моделей разного типа и пространственной детализации - от регрессионно-статистической модели YieldStat для региона (13) до динамической экофизиологической модели MONICA в пределах конкретного сельскохозяйственного поля $(31,53)$.

Еще один аспект пространственной экспансии точечных моделей продуктивности культур (29) в системы регионального планирования связан с инструментами интеграции таких моделей с ГИС $(34,36,45,54)$. Достаточно сказать, что к настоящему времени практически все наиболее распространенные модели продукционного процесса имеют специальные оболочки или расширения для организации расчетов в пространственном разрешении, например платформа CRAFT для семейства моделей DSSAT (37). Для постпроцессинга (геостатистического и пространственного анализа при моделировании процессов в посевах культур) создана Географическая информационная система по сельскому хозяйству и окружающей среде (AEGIS; AEGIS/WIN), связывающая модели DSSAT с инструментами географического картографирования ArcInfo и ArcView с помощью объекториентированного языка макропрограммирования (37). Модель ЕРІС на основе ГИС (GEPIC) - это еще один специализированный пространственный инструмент, который объединяет биофизическую модель ЕPIC (Environmental Policy Integrated Climate) с ГИС для моделирования пространственной и временно́й динамики основных процессов в системе почварастение-атмосфера (55). Еще в 1990-х годах в рамках проекта MARS, координируемого Объединенным исследовательским центром (Joint Research Centre - JRC, European Commission, https://ec.europa.eu/jrc/en), были разработаны Европейская система мониторинга роста сельскохозяйственных культур (https://ec.europa.eu/jrc/en/research-topic/agricultural-monitoring) и система прогнозирования урожайности на базе модели BioMA (56). В peзультате модели роста и развития растений стали совместимыми с данными дистанционного зондирования и ГИС $(5,18,19,57)$. Среди других решений этого класса можно также упомянуть продукты pSIMS, MINK, SIMPLACE и GeoSIM (58-61).

Специальная задача состоит в том, чтобы использовать в динамических моделях агроэкосистем данные дистанционного зондирования сельскохозяйственных угодий $(10,14,39,41,44,62)$. С появлением дистанционных и даже космических средств мониторинга оказалось, что многие характеристики состояния системы почва-растение-атмосфера сегодня допускают прямое оперативное измерение с практически произвольно задаваемым временным и пространственным разрешением. Причем для этого зачастую не требуется никаких особых усилий или дорогостоящих изме- 
рений, достаточно иметь доступ к частично или полностью открытым и оперативно обновляющимся базам данных обработки снимков дистанционного спутникового зондирования. Проблема, однако, заключается в том, что, несмотря на развитие средств измерения, с математической точки зрения большинство агроэкологических моделей все еще представляют собой ненаблюдаемые системы управления (63), в которых оценке по имеющимся наблюдениям доступна лишь малая часть переменных состояния. Более того, большинство соответствующих наблюдений представляют собой измерения косвенных величин - неких оптических индексов, которые, безусловно, связаны с сущностными переменными модели (биомассой побега, влажностью почвы, содержанием хлорофилла, уровнем азотного питания и т.д.), но связь эта часто неоднозначна и слабо формализуема (64).

Анализ современного состояния проблемы показывает, что имитационное моделирование агроэкосистем - это технология, которая основана на результатах специальных исследований (54) и в настоящее время применяется во многих областях, таких как прогнозирование урожайности (65), изменений климата $(29,65,66)$, реакции культур при различных обработках и в зависимости от внешних условий (67). В самое ближайшее время ожидается появление новых приложений с использованием этих моделей для целей точного земледелия и интеллектуального сельского хозяйства (Smart Agriculture). Признанные мировые лидеры в этой области DSSAT (68) и EPIC (69) (США), STICS (70) (Франция), APSIM (54) (Австралия), AGROTOOL (29) (Россия), MONICA (31) (Германия) и WOFOST (65) (Нидерланды). География практического использования имитационных моделей агроэкосистем также широка $(52,71)$. При этом, как и в других приложениях наук о Земле (климатология, гидрология), здесь наблюдается устойчивая тенденция использовать для решения конкретных задач не одну, а несколько моделей (ансамблевые расчеты), тем более что многие из них имеются в свободном доступе $(72,73)$.

Однако следует отметить, что имитационные модели все еще мало применяются в приложениях, использующих долгосрочные прогнозы (54, 74), то есть для разработки систем земледелия и землепользования, хотя есть и положительные примеры $(75,76)$. Основным вызовом остается необходимость осуществлять непрерывный расчет динамики состояний агроэкосистемы для выбранной географической локации с учетом смены культур и межвегетационных периодов. Иногда для этого полное описание севооборота непосредственно включается во входные данные (пример - модель MONICA) (31), альтернативный подход - специальные платформы и оболочки для автоматизированного переноса состояния моделируемого объекта от предшествующего запуска модели к последующему (76). Многие системы пространственного моделирования культур были разработаны для конкретных применений и, следовательно, имеют определенные требования и ограничения, которые могут существенно затруднить их внедрение в конкретных регионах. Из таких инструментов одни уже устарели (AEGIS) (77), другие очень надежны (pSIMS, Mink) (59), в них используются языки сценариев, и расчеты в этих системах должны выполняться на вычислительных кластерах или на высокопроизводительных компьютерах. С некоторыми моделями (MARS) (21) возникают трудно разрешимые проблемы, связанные с практикой применения и обслуживанием (71), а некоторые решения ограничены по функционалу (59). При этом все исследователи указывают на необходимость удобной и простой в использовании программной платформы, которая могла бы стать доступной и адаптируемой средой расчета базовых моделей продукционного процесса для облегчения прогнозиро- 
вания роста и развития растений при разных региональных системах земледелия $(39,54,78,79)$.

Практические примеры оперативного и долгосрочного прогнозирования продуктивности посевов. Идеализированная масштабируемая информационно-аналитическая система динамического мониторинга и прогнозирования характеристик состояния сельскохозяйственной территории должна использоваться для разработки систем земледелия на разных уровнях пространственной и временно́й детализации (80). По пространственному разрешению эти задачи могут быть разделены на три группы. Первая - макромасштабные расчеты: оценка потенциальной и мониторинг достижимой продуктивности основных сельскохозяйственных культур в масштабе страны для текущих условий (оперативное управление по времени) и возможных климатических изменений (стратегическое планирование во времени). Вторая группа - мезомасштабные расчеты: мониторинг продуктивности и экологической устойчивости агроландшафтов (оперативное управление), а также модельный анализ и оптимизация систем земледелия в масштабе региона (стратегическое управление). Наконец, третья труппа - микромасштабные расчеты: анализ (как оперативный, так многолетний) эффективности технологий точного или координатного земледелия в растениеводческом хозяйстве или на конкретном поле. Представление о типах задач, которые могут быть поставлены и решены с помощью массовых расчетов точечных динамических моделей агроэкосистемы в различном временном и пространственном масштабе, а также о методах создания универсальной среды для таких расчетов, дает таблица 1 (81).

1. Задачи, решаемые с помощыю имитационной модели агроэкосистемы на различном пространственном (ПУУ) и временно́м (ВУУ) уровне управления (81)

\begin{tabular}{|c|c|c|c|}
\hline Bуy & $\begin{array}{c}\text { Микроуровень } \\
\text { (фермерское } \\
\text { хозяйство, поле) }\end{array}$ & $\begin{array}{c}\text { Мезоуровень } \\
\text { (регион, область, агрохолдинг) }\end{array}$ & $\begin{array}{c}\text { Макроуровень } \\
\text { (страна, континент) }\end{array}$ \\
\hline $\begin{array}{l}\text { Оперативный мониторинг } \\
\text { и прогноз }\end{array}$ & $\begin{array}{l}\text { Поддержка оператив- } \\
\text { ных решений в точ- } \\
\text { ном земледелии } \\
\text { Ассимиляция данных }\end{array}$ & \multicolumn{2}{|c|}{$\begin{array}{l}\text { Оперативно уточняющийся прогноз ожидаемой продук- } \\
\text { тивности в ходе текущего сезона вегетации }\end{array}$} \\
\hline $\begin{array}{l}\text { Многолетний анализ } \\
\text { и планирование }\end{array}$ & $\begin{array}{l}\text { Проекты землеустрой- } \\
\text { ства на уровне фермы }\end{array}$ & $\begin{array}{l}\text { Проектирование региональных } \\
\text { систем земледелия }\end{array}$ & $\begin{array}{l}\text { климатических } \\
\text { изменений на сель- } \\
\text { ское хозяйство }\end{array}$ \\
\hline & & $\begin{array}{l}\text { Стратегический анализ последстви } \\
\text { новых технологий и интродукции }\end{array}$ & $\begin{array}{l}\text { Iй внедрения } \\
\text { новых культур }\end{array}$ \\
\hline
\end{tabular}

Массовые расчеты модели по представительному набору пространственных точек должны проводиться в рамках общей идеологии пакетного расчета всех сценариев сформированного проекта вычислительного эксперимента, где принадлежность сценария к конкретной точке определяется соответствующими градациями факторов «почва» и «местность» $(82,83)$. В таблице 2 перечислены соответствующие источники и механизмы оперативного пополнения данных при расчетах различной пространственной и временно́й детализации (83).

2. Источники и типы входных данных для моделирования продукционного процесса агроэкосистем с различной пространственной детализацией (83)

\begin{tabular}{|c|c|c|c|}
\hline$\overbrace{\text { Фактор }}$ ПУ & $\begin{array}{l}\text { Микроуровень } \\
\text { (агрохозяйство) }\end{array}$ & $\begin{array}{c}\text { Мезоуровень } \\
\text { (географический регион) }\end{array}$ & $\begin{array}{c}\text { Макроуровень } \\
\text { (страна, континент) }\end{array}$ \\
\hline Почва & $\begin{array}{l}\text { Детальные данные по } \\
\text { почвенным разрезам }\end{array}$ & $\begin{array}{l}\text { Почвенные карты с привяз- } \\
\text { кой кадастровых данных }\end{array}$ & $\begin{array}{l}\text { Почвенные карты статисти- } \\
\text { ческого характера }\end{array}$ \\
\hline Местоположение & $\begin{array}{l}\text { Координаты и цифровые } \\
\text { карты рельефа }\end{array}$ & Координаты & Координаты \\
\hline
\end{tabular}


Погода
Оперативное управление: точное земледелие

Стратегическое планирование автоматизированный подбор технологий

Конкретные культуры и сорта Культуры в рамках анализитекущего сезона севооборота

Оперативное управление: локальная автоматическая метеостанция

Стратегическое планирование: одноточечный генератор погоды, откалиброванный по данным ближайшей метеостанции система земледелия ские прогнозы генератор погоды
Продолжение таблицы 2

Регионально адаптированная

Регионально адаптированные нормативные системы земледелия

Базовые сельскохозяйственные культуры

Оперативное управление: сеть опорных метеостанций и краткосрочные синоптические прогнозы и краткосрочные синоптиче-

Стратегическое планирование: Стратегическое планирование: пространственно-временной набор эталонных лет-анало-

П р и м е ч а и е. ПУ - пространственный уровень.

Для расчетов регионального масштаба важно определить набор контрольных (базовых) точек, где будут выполняться расчеты по модели, которые должны принадлежать к реальным сельскохозяйственным полям и в достаточной степени отражать разнообразие почвенных и климатических условий рассматриваемого региона. В региональных расчетах разумно использовать не конкретные, а условные, наиболее типичные для региона почвенные характеристики (например, из Единого государственного реестра почвенных ресурсов России, http://egrpr.esoil.ru/). В модель назначается не конкретный известный вариант, а усредненная технология из рекомендованных систем земледелия. Имеется несколько переменных, значения которых определяют начальное состояние агроэкосистемы, к которым очень чувствительны динамические модели во время запуска (84, 85). Поэтому следует начинать моделирование текущего сезона не с даты сева, а с достаточно продолжительного периода подготовки (76). Кроме того, для любого географического местоположения можно получить временные ряды по фактически наблюдаемой погоде с ресурсов Всемирной метеорологической ассоциации (World Meteorological Organization, WMO, Швейцария), а также краткосрочный прогноз погоды (https://www.worldweatheronline.com, https://www.aerisweather.com), по крайней мере на 3-5 сут $(39,41,86)$. При этом для прогнозного моделирования требуется большое число возможных реализаций погодных сценариев, которыми могут быть так называемые годы-аналоги (87) или стохастические генераторы погоды (88-90), теоретические аспекты применения которых разработаны и на практике они успешно интегрированы в компьютерные модели культур. В региональном масштабе можно говорить о «хорошем сезоне» и «плохом сезоне» для растениеводства, что позволяет считать однородным регионом сельскохозяйственного производства такой географический район, где межгодовая изменчивость продуктивности значительно превышает пространственную изменчивость внутри региона (80).

В России разработан прототип информационно-аналитической системы для модельного прогноза продуктивности посевов в разном пространственно-временном охвате. В качестве основного инструмента автоматизации массовых расчетов моделей продукционного процесса использовано программное обеспечение APEX (Automation of Polivariant EXperiments - автоматизация поливариантных экспериментов) $(32,42,91)$. Здесь поливариантный анализ означает проектирование и подготовку многомерного компьютерного тематического исследования, выполнение прогонов модели в пакетном режиме и применение передовых процедур статистической обработки полученных результатов.

Концепция использования массовых расчетов динамической модели 
агроэкосистем для целей оперативного прогнозирования, проактивного управления и ассимиляции данных спутникового зондирования была теоретически разработана в серии исследований $(29,42,64,75,76,80,92)$ и апробирована, в частности, на портале «Карта Урожая» (https://cropmap.ru/), который представляет собой электронную карту со встроенными сервисами мониторинга и прогноза продукционного процесса сельскохозяйственных культур, а для прогнозирования урожайности заданной культуры в выбираемой пользователем географической локации служит динамическая модель агроэкосистемы AGROTOOL $(28,29)$.

Исследование потенциальной урожайности основных сельскохозяйственных культур на всей территории Российской Федерации на базе динамического (оперативно уточняющегося) прогноза продуктивности в рамках одного текущего сезона вегетации с представлением результатов в среде ГИС может служить примером решения задачи пространственной детализации, противоположной рассмотренным выше (42). Избранные результаты моделирования иллюстрирует рисунок 1. Апробированный подход позволяет анализировать эволюцию ожиданий относительно будущей доходности в течение вегетационного периода для всех базовых точек вместе (тематические карты прогнозного среднего урожая в правой части рисунка), а также динамику и интервал прогнозируемой средней урожайности для любой конкретной базовой точки (в левой части рисунка представлен график для тестовой точки в Оренбургской области).

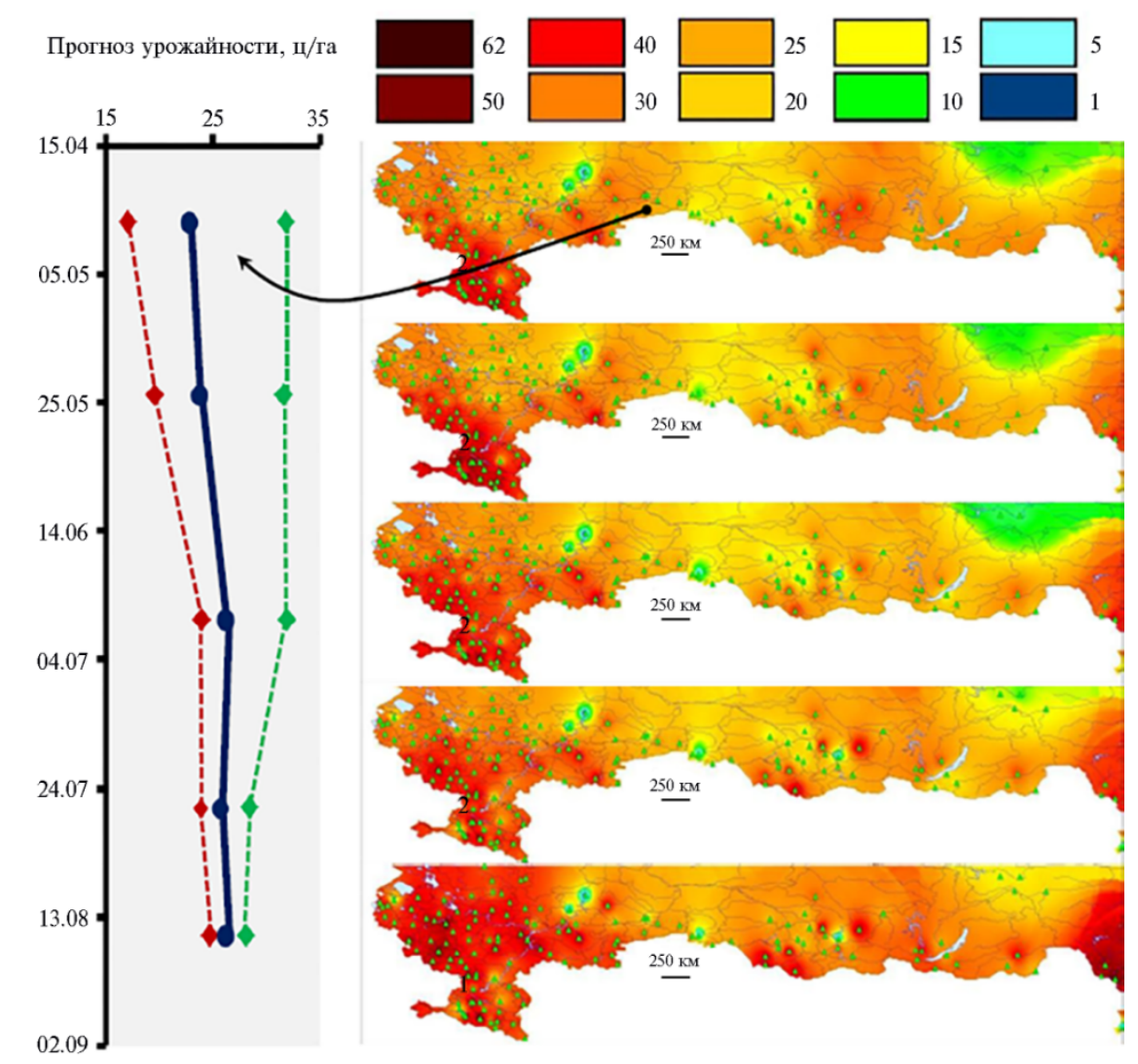

Рис. 1. Динамически скорректированный прогноз урожайности яровой пшеницы в разные даты в сезоне вегетации в 2017 году для конкретного местоположения (левая часть) и в масштабе всей России (правая часть, средние значения). Зеленые треугольники - опорные точки, в которых проводился расчет (42). 
В качестве примера успешного применения оперативного и долгосрочного прогнозирование продуктивности посевов на основе массовых расчетов имитационной модели агроэкосистемы в геоинформационной среде можно привести расчеты для Республики Крым, где исследуемой культурой был яровой ячмень. В качестве источников исходной информации использовалась оцифрованная электронная карта почв Крыма и сценарии фактических реализаций погоды по данным записей 15 крымских метеостанций сети WMO. Расчеты для сезонов вегетации 2012 и 2014 годов проводились по сетке выбранных 55 реперных точек, расположенных на полях сельскохозяйственных предприятий всех районов республики. Пространственное распределение урожайности ячменя и даты его созревания (указано число точек моделирования с той же датой созревания ячменя) представлены на рисунке 2 (80).

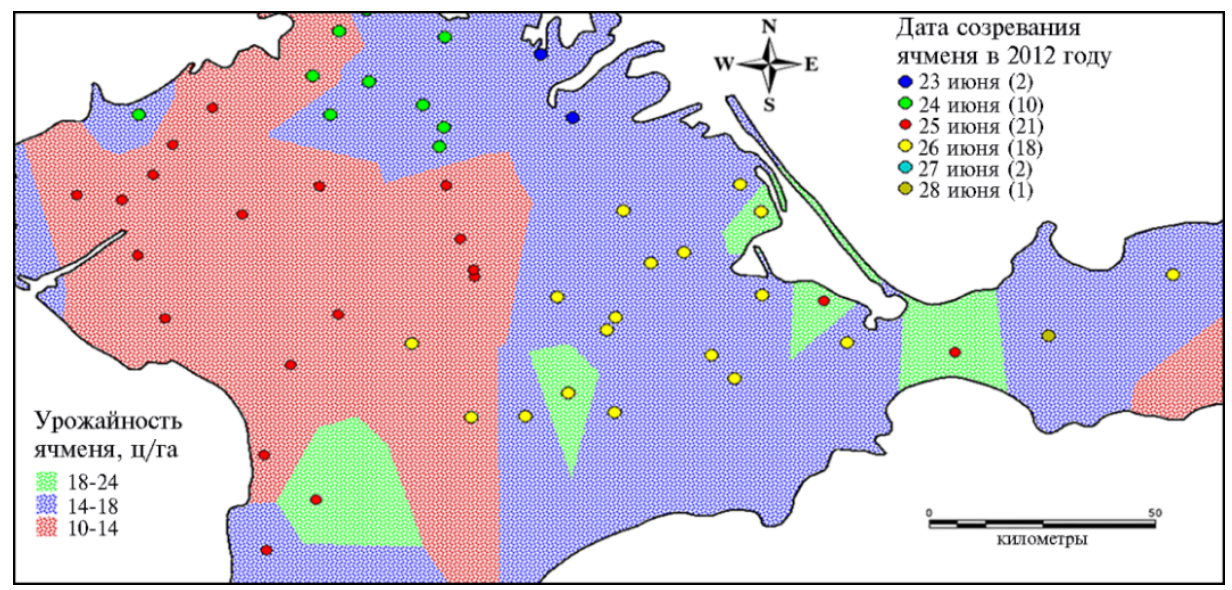

Рис. 2. Пространственное распределение урожайности ячменя и даты его созревания (в скобках число точек моделирования с одинаковой датой созревания) (80).

Также отметим ряд исследований, где методика поливариантного анализа динамики агроэкосистем на основе массовых расчетов динамических моделей продуктивности применялась в задачах долгосрочного планирования $(25,76)$. Среди наиболее интересных стоит упомянуть проект Европейского сообщества «Crop growth and soil processes modeling - the use of multi-model ensemble for crop rotations under recent and future climatic conditions» (93-95) по применению моделей продукционного процесса для оценки возможного влияния глобальных климатических изменений на продуктивность и стабильность агроэкосистем Восточной Европы, а также поиска и анализа способов смягчения этих негативных последствий. В качестве инструмента исследования выбирали не одну конкретную модель, а использовали модную современную методологию ансамблевых расчетов (96). Принципиальным отличием упомянутого проекта от многочисленных аналогов выступал тот факт, что соответствующие ансамблевые расчеты выполнялись в разрезе выбранных схем севооборота, а модификация традиционных практик смены культур рассматривалась в качестве основного исследуемого механизма, обеспечивающего смягчение последствий изменения климата для сельского хозяйства (97-99).

Таким образом, современные вычислительные и информационные технологии создают условия для поддержки оперативных агротехнологических решений, используя более точные и подробные динамические модели. Вариативное моделирование позволяет изучать эффекты различных агротехнологий и ассимилировать данные дистанционного зондирования сельско- 
хозяйственных территорий для выбора оптимального агротехнического плана и корректировки моделирования по результатам реальных измерений в течение вегетационного периода. Число вариантов расчета и соответствующих запусков модели определяется варьированием следующих определяющих факторов: пространственный фактор (расчеты проводятся в определенных местоположениях, каждое из которых характеризуется свойствами почвы и параметрами моделируемой сельхозкультуры); метеорологический фактор (возможная динамика погоды за оставшийся период вегетационного периода моделируется с использованием «вариантов возможных траекторий», состоящих из репрезентативного числа синтетических погодных сценариев, а для их формирования следует использовать стохастический генератор погоды, поддерживающего как временные, так и пространственные корреляции); технологический фактор (для обоснованного выбора даты и качества/количества технологических операций необходимо проанализировать влияние различных вариантов, чтобы выбрать лучший в контексте выбранного критерия статистической оптимизации и реализовать стратегию проактивного управления в растениеводстве); модельный фактор (желательно использовать не одну, а несколько альтернативных моделей агроэкосистем для получения достоверных результатов).

Одновременное варьирование перечисленных выше факторов с набором их градаций приводит к тому, что общее число вариантов одного прогона модели, реализующих полный факторный вычислительный эксперимент, определяет необходимость использовать современные технологии распределенных параллельных вычислений и суперкомпьютерную технику. Анализ сложившейся ситуации проектировании систем земледелия позволяет определить важнейшую роль и потенциальную востребованность в этой сфере динамических моделей агроэкосистем. Лица, принимающие решения, в идеальной ситуации желали бы иметь инструмент, который на основе самых разнородных источников информации, включая данные дистанционного зондирования, дает возможность оценить последствия управленческих решений по выбору систем земледелия на среднесрочную и долгосрочную перспективу. Имитационные модели агроэкосистем, работающие совместно с ГИС, представляют собой наилучшую среду для решения оеалтзации сценариев «что будет, если...». Подобный подход может стать мощнейшим инструментом при оптимизации использования земель сельскохозяйственного назначения. Создание адекватной информационно-аналитической системы позволяет производителям сельскохозяйственной продукции, а также планирующим, контролирующим и регулирующим инстанциям принимать решения на ином качественном уровне. Такой информационный сервис может использоваться как внешний интеллектуальный компонент государственных информационно-аналитических ресурсов в рамках принятой Национальной платформы цифрового государственного управления сельским хозяйством «Цифровое сельское хозяйство».

Итак, оперативное и долгосрочное прогнозирование продуктивности посевов на основе массовых расчетов имитационной модели агроэкосистемы в геоинформационной среде играет важнейшую роль в разработке систем земледелия. Эмпирические регрессионные модели, основанные на статистической информации, по-прежнему широко применяются для прогнозных оценок продуктивности агроэкосистем в масштабах региона. Однако их основной недостаток - относительно невысокая точность результатов, что делает невозможным использование этого подхода в задачах поддержки оперативных агротехнологических решений. Развитие вычислительных и информационных технологий позволяет ответить на этот вызов, 
применяя более точные и подробные динамические модели агроэкосистем. Остаются актуальными задачи по выбору оптимального агротехнического плана и корректировка процесса моделирования в режиме онлайн на основе результатов реальных измерений в течение вегетационного периода. При их решении основными инструментами служат вариативное моделирование для анализа эффектов различных модификаций агротехнологий и ассимиляция данных дистанционного зондирования сельскохозяйственных территорий, а наилучшей средой - имитационные модели агроэкосистем, работающие совместно с ГИС.

\section{ЛИТЕРАТУРА}

1. Rodriguez D., de Voil P., Rufino M.C., Odendo M., Van Wijk M.T. To mulch or to munch? Big modelling of big data. Agricultural Systems, 2017, 153: 32-42 (doi: 10.1016/j.agsy.2017.01.010).

2. Sierra J., Causeret F., Chopin P. A framework coupling farm typology and biophysical modelling to assess the impact of vegetable crop-based systems on soil carbon stocks. Application in the Caribbean. Agricultural Systems, 2017, 153: 172-180 (doi: 10.1016/j.agsy.2017.02.004).

3. Jeuffroy M.H., Casadebaig P., Debaeke P., Loyce C., Meynard J.M. Agronomic model uses to predict cultivar performance in various environments and cropping systems. A review. Agronomy for Sustainable Development, 2014, 34(1): 121-137. (doi: 10.1007/s13593-013-0170-9).

4. Dury J., Schaller N., Garcia F., Reynaud A., Bergez J. E. Models to support cropping plan and crop rotation decisions. A review. Agronomy for Sustainable Development, 2012, 32(2): 567-580. (doi: 10.1007/s13593-011-0037-x).

5. Jones J.W., Antle J.M., Basso B., Boote K.J., Conant R.T., Foster I., Godfray H.C.J., Herrero M., Howitt R.E., Janssen S., Keating B.A., Munoz-Carpena R., Porter C.H., Rosenzweig C., Wheeler T.R. Brief history of agricultural systems modeling. Agricultural Systems, 2017, 155: 240-254 (doi: 10.1016/j.agsy.2016.05.014).

6. Morais R., Silva N., Mendes J., Adão T., Pádua L., Lypez-Riquelme J.A., Pavón-Pulido N., Sousa J.J., Peres E. Mysense: A comprehensive data management environment to improve precision agriculture practices. Computers and Electronics in Agriculture, 2019, 162: 882-894 (doi: 10.1016/j.compag.2019.05.028).

7. Insua J.R., Utsumi S.A., Basso B. Estimation of spatial and temporal variability of pasture growth and digestibility in grazing rotations coupling unmanned aerial vehicle (UAV) with crop simulation models. PloS ONE, 2019, 14(3): e0212773 (doi: 10.1371/journal.pone.0212773).

8. Gebremedhin A., Badenhorst P.E., Wang J., Spangenberg G.C., Smith K.F. Prospects for measurement of dry matter yield in forage breeding programs using sensor technologies. Agronomy, 2019, 9(2): 65 (doi: 10.3390/agronomy9020065).

9. Reynolds D., Ball J., Bauer A., Davey R., Griffiths S., Zhou J. CropSight: a scalable and opensource information management system for distributed plant phenotyping and IoT-based crop management. GigaScience, 2019, 8(3): giz009 (doi: 10.1093/gigascience/giz009).

10. Hunt E.R. Jr., Daughtry C.S. What good are unmanned aircraft systems for agricultural remote sensing and precision agriculture? International Journal of Remote Sensing, 2018, 39(15-16): 53455376 (doi: 10.1080/01431161.2017.1410300).

11. Farooque A.A., Chang Y.K., Zaman Q.U., Groulx D., Schuman A.W., Esau T.J. Performance evaluation of multiple ground-based sensors mounted on a commercial wild blueberry harvester to sense plant height, fruit yield and topographic features in real-time. Computers and Electronics in Agriculture, 2013, 91: 135-144 (doi: 10.1016/j.compag.2012.12.006).

12. Sankaran S., Khot L.R., Espinoza C.Z., Jarolmasjed S., Sathuvalli V.R., Vandemark G.J., Miklas P.N., Carter A.H., Pumphrey M.O., Knowles N.R.N., Pavek M.J. Low-altitude, high-resolution aerial imaging systems for row and field crop phenotyping: A review. European Journal of Agronomy, 2015, 70: 112-123 (doi: 10.1016/j.eja.2015.07.004).

13. Mirschel W., Wieland R., Wenkel K.O., Nendel C., Guddat C. YIELDSTAT - a spatial yield model for agricultural crops. European Journal of Agronomy, 2014, 52: 33-46 (doi: 10.1016/j.eja.2013.09.015).

14. Kern A., Barcza Z., Marjanovi H., Árendás T., Fodor N., Bynis P., Bognár P., Lichtenberger J. Statistical modelling of crop yield in Central Europe using climate data and remote sensing vegetation indices. Agricultural and Forest Meteorology, 2018, 260: 300-320 (doi: 10.1016/j.agrformet.2018.06.009).

15. Conradt T., Gornott C., Wechsung F. Extending and improving regionalized winter wheat and silage maize yield regression models for Germany: enhancing the predictive skill by panel definition through cluster analysis. Agricultural and Forest Meteorology, 2016, 216: 68-81 (doi: 10.1016/j.agrformet.2015.10.003).

16. Gutzler C., Helming K., Balla D., Dannowski R., Deumlich D., Glemnitz M., Sieber S. Agricultural land use changes - a scenario-based sustainability impact assessment for Brandenburg, 
Germany. Ecological Indicators, 2015, 48: 505-517 (doi: 10.1016/j.ecolind.2014.09.004).

17. Grados D., Schrevens E. Multidimensional analysis of environmental impacts from potato agricultural production in the Peruvian Central Andes. Science of the Total Environment, 2019, 663: 927-934 (doi: 10.1016/j.scitotenv.2019.01.414).

18. Antle J.M., Jones J.W., Rosenzweig C. Next generation agricultural system models and knowledge products: synthesis and strategy. Agricultural Systems, 2017, 155: 179-185 (doi: 10.1016/j.agsy.2017.05.006).

19. Janssen S.J., Porter C.H., Moore A.D., Athanasiadis I.N., Foster I., Jones J.W., Antle J.M. Towards a new generation of agricultural system data, models and knowledge products: information and communication technology. Agricultural Systems, 2017, 155: 200-212 (doi: 10.1016/j.agsy.2016.09.017).

20. Fielke S., Taylor B., Jakku E. Digitalisation of agricultural knowledge and advice networks: a state-of-the-art review. Agricultural Systems, 2020, 180: 102763 (doi: 10.1016/j.agsy.2019.102763).

21. Lecerf R., Ceglar A., Lypez-Lozano R., Van Der Velde M., Baruth B. Assessing the information in crop model and meteorological indicators to forecast crop yield over Europe. Agricultural Systems, 2019, 168: 191-202 (doi: 10.1016/j.agsy.2018.03.002).

22. Ozturk I., Sharif B., Baby S., Jabloun M., Olesen J.E. The long-term effect of climate change on productivity of winter wheat in Denmark: a scenario analysis using three crop models. The Journal of Agricultural Science, 2017, 155(5): 733-750 (doi: 10.1017/S0021859616001040).

23. Hannah L., Donatti C.I., Harvey C.A., Alfaro E., Rodriguez D.A., Bouroncle C., Castellanos E., Diaz F., Fung E., Hidalgo H.G., Imbach P. Regional modeling of climate change impacts on smallholder agriculture and ecosystems in Central America. Climatic Change, 2017, 141(1): 2945 (doi: 10.1007/s10584-016-1867-y).

24. Belem M., Saqalli M. Development of an integrated generic model for multi-scale assessment of the impacts of agro-ecosystems on major ecosystem services in West Africa. Journal of Environmental Management, 2017, 202: 117-125 (doi: 10.1016/j.jenvman.2017.07.018).

25. Badenko V.L., Topaj A.G., Yakushev V.V., Mirschel W., Nendel C. Crop models as research and interpretative tools. Sel'skokhozyaistvennaya Biologiya, 2017, 52: 437-445 (doi: 10.15389/agrobiology.2017.3.437eng).

26. Anten N.P., Vermeulen P.J. Tragedies and crops: understanding natural selection to improve cropping systems. Trends in Ecology \& Evolution, 2016, 31(6): 429-439 (doi: 10.1016/j.tree.2016.02.010).

27. Lee H., Lautenbach S., Nieto A.P.G., Bondeau A., Cramer W., Geijzendorffer I.R. The impact of conservation farming practices on Mediterranean agro-ecosystem services provisioning - a meta-analysis. Regional Environmental Change, 2019, 19: 2187-2202 (doi: 10.1007/s10113-0181447-y).

28. Poluektov R.A., Fintushal S.M., Oparina I.V., Shatskikh D.V., Terleev V.V., Zakharova E.T. AGROTOOL - a system for crop simulation. Archives of Agronomy and Soil Science, 2002, 48(6): 609-635 (doi: 10.1080/0365034021000041597).

29. Badenko V.L., Terleev V.V., Topaj A. G. AGROTOOL software as an intellectual core of decision support systems in computer aided agriculture. Applied Mechanics and Materials, 2014, 635-637: 1688-1691 (doi: 10.4028/www.scientific.net/AMM.635-637.1688).

30. de Wit C.T., Van Keulen H. Modelling production of field crops and its requirements. Geoderma, 1987, 40(3-4): 253-265 (doi: 10.1016/0016-7061(87)90036-X).

31. Nendel C., Kersebaum K.C., Mirschel W., Wenkel K.O. Testing farm management options as climate change adaptation strategies using the MONICA model. European Journal of Agronomy, 2014, 52: 47-56 (doi: 10.1016/j.eja.2012.09.005).

32. Medvedev S., Topaj A. Crop simulation model registrator and polyvariant analysis. IFIP Advances in Information and Communication Technology, 2011, 359: 295-301 (doi: 10.1007/978-3-64222285-6_32).

33. Ramírez-Cuesta J.M., Mirás-Avalos J.M., Rubio-Asensio J.S., Intrigliolo D.S. A novel ArcGIS toolbox for estimating crop water demands by integrating the dual crop coefficient approach with multi-satellite imagery. Water, 2019, 11(1): 38 (doi: 10.3390/w11010038)

34. Liben F.M., Wortmann C.S., Tirfessa A. Geospatial modeling of conservation tillage and nitrogen timing effects on yield and soil properties. Agricultural Systems, 2020, 177: 102720 (doi: 10.1016/j.agsy.2019.102720).

35. Liben F.M., Wortmann C.S., Yang H., Lindquist J.L., Tadesse T., Wegary D. Crop model and weather data generation evaluation for conservation agriculture in Ethiopia. Field Crops Research, 2018, 228: 122-134 (doi: 10.1016/j.fcr.2018.09.001).

36. Hartkamp A.D., White J.W., Hoogenboom G. Interfacing geographic information systems with agronomic modeling: a review. Agronomy Journal, 1999, 91(5): 761-772 (doi: 10.2134/agronj1999.915761x).

37. Shelia V., Hansen J., Sharda V., Porter C., Aggarwal P., Wilkerson C.J., Hoogenboom G. A multi-scale and multi-model gridded framework for forecasting crop production, risk analysis, and climate change impact studies. Environmental Modelling \& Software, 2019, 115: 144-154 (doi: 10.1016/j.envsoft.2019.02.006).

38. Bassoa B., Liua L. Seasonal crop yield forecast: methods, applications, and accuracies. Advances in Agronomy, 2018, 154: 201-255 (doi: 10.1016/bs.agron.2018.11.002). 
39. Huang J., Gómez-Dans J., Huang H., Ma H., Wu Q., Lewis P., Liang S., Chen Z., Xue J., Wu Y., Zhao F., Wang J., Xie X. Assimilation of remote sensing into crop growth models: Current status and perspectives. Agricultural and Forest Meteorology, 2019, 276: 107-109 (doi: 10.1016/j.agrformet.2019.06.008).

40. Machwitz M., Hass E., Junk J., Udelhoven T., Schlerf M. Crop GIS - a web application for the spatial and temporal visualization of past, present and future crop biomass development. Computers and Electronics in Agriculture, 2019, 161: 185-193 (doi: 10.1016/j.compag.2018.04.026).

41. Jin X., Kumar L., Li Z., Feng H., Xu X., Yang G., Wang, J. A review of data assimilation of remote sensing and crop models. European Journal of Agronomy, 2018, 92: 141-152 (doi: 10.1016/j.eja.2017.11.002).

42. Баденко В.Л., Топаж А.Г., Медведев С.А., Захарова Е.Т. Оперативно уточняющийся прогноз урожайности пшеницы в сельскохозяйственных зонах на всей территории России на базе имитационной модели продуктивности. АгроЭкоИнфо, 2018, 3(33): 68.

43. Reynolds M., Kropff M., Crossa J., Koo J., Kruseman G., Molero Milan A., Rutkoski J., Schulthess U., Balwinder-Singh, Sonder K., Tonnang H., Vadez V. Role of modelling in international crop research: overview and some case studies. Agronomy, 2018, 8(12): 291 (doi: 10.3390/agronomy8120291).

44. Kumhálová J., Matějková Š. Yield variability prediction by remote sensing sensors with different spatial resolution. International Agrophysics, 2017, 31(2): 195-202 (doi: 10.1515/intag-2016-0046).

45. Resop J.P., Fleisher D.H., Wang Q., Timlin D.J., Reddy V.R. Combining explanatory crop models with geospatial data for regional analyses of crop yield using field-scale modeling units. Computers and Electronics in Agriculture, 2012, 89: 51-61 (doi: 10.1016/j.compag.2012.08.001).

46. Hodson D., White J. GIS and crop simulation modelling applications in climate change research. In: Climate change and crop production. CABI Publishers, Wallingford, UK, 2010: 245-262 (doi: 10.1079/9781845936334.0245).

47. Akpoti K., Kabo-bah A.T., Zwart S.J. Agricultural land suitability analysis: state-of-the-art and outlooks for integration of climate change analysis. Agricultural Systems, 2019, 173: 172-208 (doi: 10.1016/j.agsy.2019.02.013).

48. Mirschel W., Schultz A., Wenkel K.O., Wieland R., Poluektov R.A. Crop growth modelling on different spatial scales - a wide spectrum of approaches. Archives of Agronomy and Soil Science, 2004, 50(3): 329-343 (doi: 10.1080/03650340310001634353).

49. Gaso D.V., Berger A.G., Ciganda V.S. Predicting wheat grain yield and spatial variability at field scale using a simple regression or a crop model in conjunction with Landsat images. Computers and Electronics in Agriculture, 2019, 159: 75-83 (doi: 10.1016/j.compag.2019.02.026).

50. Maharjan G.R., Hoffmann H., Webber H., Srivastava A.K., Weihermbller L., Villa A., Coucheney E., Lewan E., Trombi G., Moriondo M., Bindi M., Grosz B., Dechow R., Kuhnert M., Doro L., Kersebaum K.-C., Stella T., Specka X., Nendel C., Constantin J., Raynal H., Ewert F., Gaiser T. Effects of input data aggregation on simulated crop yields in temperate and Mediterranean climates. European Journal of Agronomy, 2019, 103: 32-46 (doi: 10.1016/j.eja.2018.11.001).

51. Wenkel K.O., Berg M., Mirschel W., Wieland R., Nendel C., Kustner B. LandCaRe DSS - an interactive decision support system for climate change impact assessment and the analysis of potential agricultural land use adaptation strategies. Journal of Environmental Management, 2013, 127: 168-183 (doi: 10.1016/j.jenvman.2013.02.051).

52. Zhai Z., Martínez J.F., Beltran V., Martínez N.L. Decision support systems for agriculture 4.0: Survey and challenges. Computers and Electronics in Agriculture, 2020, 170: 105256 (doi: 10.1016/j.compag.2020.105256).

53. Battisti R., Parker P.S., Sentelhas P. C., Nendel C. Gauging the sources of uncertainty in soybean yield simulations using the MONICA model. Agricultural Systems, 2017, 155: 9-18 (doi: 10.1016/j.agsy.2017.04.004).

54. Holzworth D.P., Snow V., Janssen S., Athanasiadis I.N., Donatelli M., Hoogenboom G., White J.W., Thorburn P. Agricultural production systems modelling and software: current status and future prospects. Environmental Modelling \& Software, 2015, 72: 276-286 (doi: 10.1016/j.envsoft.2014.12.013).

55. Yin Y., Zhang X., Lin D., Yu H., Shi P. GEPIC-VR model: a GIS-based tool for regional crop drought risk assessment. Agricultural Water Management, 2014, 144: 107-119 (doi: 10.1016/j.agwat.2014.05.017).

56. Donatelli M., Srivastava A.K., Duveiller G., Niemeyer S., Fumagalli D. Climate change impact and potential adaptation strategies under alternate realizations of climate scenarios for three major crops in Europe. Environmental Research Letters, 2015, 10(7): 075005 (doi: 10.1088/17489326/10/7/075005).

57. Seguini L., Bussay A., Baruth B. From extreme weather to impacts: the role of the areas of concern maps in the JRC MARS bulletin. Agricultural Systems, 2019, 168: 213-223 (doi: 10.1016/j.agsy.2018.07.003).

58. Thorp K.R., Bronson K.F. A model-independent open-source geospatial tool for managing pointbased environmental model simulations at multiple spatial locations. Environmental Modelling \& Software, 2013, 50: 25-36 (doi: 10.1016/j.envsoft.2013.09.002). 
59. Elliott J., Kelly D., Chrryssanthacopoulos J., Glotter M., Jhunjhnuwala K., Best N., Wilde M. Foster I. The parallel system for integrating impact models and sectors (pSIMS). Environmental Modelling \& Software, 2014, 62: 509-516. (doi: 10.1016/j.envsoft.2014.04.008).

60. Gabaldyn-Leal C., Webber H., Otegui M.E., Slafer G.A., Ordócez R.A., Gaiser T., Lorite I.J., Ruiz-Ramos M., Ewert F. Modelling the impact of heat stress on maize yield formation. Field Crops Research, 2016, 198: 226-237 (doi: 10.1016/j.fcr.2016.08.013)

61. Huang J., Scherer L., Lan K., Chen F., Thorp K.R. Advancing the application of a modelindependent open-source geospatial tool for national-scale spatiotemporal simulations. Environmental Modelling \& Software, 2019, 119: 374-378 (doi: 10.1016/j.envsoft.2019.07.003).

62. Calera A., Campos I., Osann A., D’Urso G., Menenti M. Remote sensing for crop water management: from ET modelling to services for the end users. Sensors, 2017, 17(5): 1104 (doi: $10.3390 / \mathrm{s} 17051104)$

63. Сучков А.П. Некоторые подходы к интеграции аналитических данных существующих и перспективных систем поддержки принятия решений. Системы и средства информатики, 2015, 25(3): 195-205 (doi: 10.14357/08696527150312).

64. Топаж А.Г., Митрофанов Е.П. Ассимиляция данных в имитационном моделировании экологических процессов методом минимизации корректирующих возмущений. Вестник Санкт-Петербургского университета. Прикладная математика. Информатика. Процессы управления, 2017, 13(3): 326-338 (doi: 10.21638/11701/spbu10.2017.309).

65. de Wit A., Boogaard H., Fumagalli D., Janssen S., Knapen R., van Kraalingen D., Supit I., van der Wijngaart R., van Diepen K. 25 years of the WOFOST cropping systems model. Agricultural Systems, 2019, 168: 154-167 (doi: 10.1016/j.agsy.2018.06.018).

66. Ewert F., Rutter R.P., Bindi M., Webber H., Trnka M., Kersebaum K.C., Olesen J.E., van Ittersum M.K., Janssen S., Rivington M., Semenov M.A., Wallach D., Porter J.R., Stewart D., Verhagen J., Gaiser T., Palosuo T., Tao F., Nendel C., Roggero P.P., Bartosová L., Asseng S. Crop modelling for integrated assessment of risk to food production from climate change. Environmental Modelling \& Software, 2015, 72: 287-303 (doi: 10.1016/j.envsoft.2014.12.003).

67. Asseng S., Ewert F., Rosenzweig C., Jones J.W., Hatfield J.L., Ruane A.C., Boote K.J., Thorburn P.J., Rötter R.P., Cammarano D., Brisson N., Basso B., Martre P., Aggarwal P.K., Angulo C., Bertuzzi P., Biernath C., Challinor A.J., Doltra J., Gayler S., Goldberg R., Grant R., Heng L., Hooker J., Hunt L.A., Ingwersen J., Izaurralde R.C., Kersebaum K.C., Müller C., Naresh Kumar S., Nendel C., O’Leary G., Olesen J.E., Osborne T.M., Palosuo T., Priesack E., Ripoche D., Semenov M.A., Shcherbak I., Steduto P., Stцckle C., Stratonovitch P., Streck T., Supit I., Tao F., Travasso M., Waha K., Wallach D., White J.W., Williams J.R., Wolf J. Uncertainty in simulating wheat yields under climate change. Nature Climate Change, 2013, 3(9): 827832 (doi: 10.1038/nclimate1916).

68. Jones J.W., Hoogenboom G., Porter C.H., Boote K.J., Batchelor W.D., Hunt L.A., Wilkens P.W., Singh U., Gijsman A.J., Ritchie J.T. The DSSAT cropping system model. European Journal of Agronomy, 2003, 18(3-4): 235-265 (doi: 10.1016/S1161-0301(02)00107-7).

69. Wang X., Williams J.R., Gassman P.W., Baffaut C., Izaurralde R.C., Jeong J., Kiniry J.R. EPIC and APEX: model use, calibration, and validation. Transactions of the ASABE, 2012, 55(4): 14471462 (doi: 10.13031/2013.42253).

70. Brisson N., Gary C., Justes E., Roche R., Mary B., Ripoche D., Zimmer D., Sierraa J., Bertuzzi P., Burgera P., Bussière F., Cabidoche Y.M., Cellier P., Debaeke P., Gaudillère J.P., Hénault C., Maraux F., Seguin B., Sinoquet H. An overview of the crop model STICS. European Journal of Agronomy, 2003, 18(3-4): 309-332 (doi: 10.1016/S1161-0301(02)00110-7).

71. Leghari S.J., Hu K., Liang H., Wei Y. Modeling water and nitrogen balance of different cropping systems in the North China Plain. Agronomy, 2019, 9(11): 696 (doi: 10.3390/agronomy9110696).

72. Jiang R., He W., Zhou W., Hou Y., Yang J.Y., He P. Exploring management strategies to improve maize yield and nitrogen use efficiency in northeast China using the DNDC and DSSAT models. Computers and Electronics in Agriculture, 2019, 166: 104-988 (doi: 10.1016/j.compag.2019.104988).

73. Xiang Z., Bailey R.T., Nozari S., Husain Z., Kisekka I., Sharda V., Gowda P. DSSAT-MODFLOW: a new modeling framework for exploring groundwater conservation strategies in irrigated areas. Agricultural Water Management, 2020, 232: 106033 (doi: 10.1016/j.agwat.2020.106033).

74. Newbery F., Qi A., Fitt B.D. Modelling impacts of climate change on arable crop diseases: progress, challenges and applications. Current Opinion in Plant Biology, 2016, 32: 101-109 (doi: 10.1016/j.pbi.2016.07.002).

75. Баденко В.Л., Гарманов В.В., Иванов Д.А., Савченко А.Н., Топаж А.Г. Перспективы использования динамических моделей агроэкосистем в задачах средне- и долгосрочного планирования сельскохозяйственного производства и землеустройства. Доклады Российской академии сельскохозяйственных наук, 2015, 1-2: 72-76.

76. Badenko V.L., Badenko G., Topaj A.G., Medvedev S., Zakharova E., Terleev V.V. Comparative simulation of various agricultural land use practices for analysis of impacts on environments. Environments, 2017, 4(4): 92 (doi: 10.3390/environments4040092).

77. Engel T., Hoogenboom G., Jones J.W., Wilkens P.W. AEGIS/WIN: a computer program for the application of crop simulation models across geographic areas. Agronomy Journal, 1997, 89(6): 
919-928 (doi: 10.2134/agronj1997.00021962008900060012x).

78. McDonald C.K., MacLeod N.D., Lisson S., Corfield J.P. The Integrated Analysis Tool (IAT) a model for the evaluation of crop-livestock and socio-economic interventions in smallholder farming systems. Agricultural Systems, 2019, 176: 102659 (doi: 10.1016/j.agsy.2019.102659).

79. Zhao G., Bryan B.A., King D., Luo Z., Wang E., Bende-Michl U., Song X., Yu Q. Large-scale, high-resolution agricultural systems modeling using a hybrid approach combining grid computing and parallel processing. Environmental Modelling \& Software, 2013, 41: 231-238 (doi: 10.1016/j.envsoft.2012.08.007).

80. Баденко В.Л., Топаж А.Г., Медведев С.А., Захарова Е.Т., Дунаева Е.А. Оценка продуктивности агроландшафтов в региональном масштабе на основе интеграции имитационной модели агроэкосистемы и ГИС. Таврический вестник аграрной науки, 2019, 3: 18-30 (doi: 10.33952/2542-0720-2019-3-19-18-30).

81. Арефьев Н.В., Баденко В.Л., Латышев, Н.К. Геоэкологические подходы к разработке информационно-аналитических систем для гидромелиоративного строительства и природообустройства. Научно-технические ведомости СПбПУ. Естественные и инженерные науки, 2010, 110: 205-210.

82. Медведев С.А., Полуэктов Р.А., Топаж А.Г. Оптимизация стратегии орошения с использованием методов поливариантного анализа динамики агроэкосистем. Мелиорация и водное хозяйство, 2012, 2: 10-13.

83. Topaj A., Badenko V., Medvedev S., Terleev V. Dynamically adjusted forecasting of agro-landscape productivity using massive computations of crop model in GIS environment. В кн.: Новые методы и результаты исследований ландшафтов в Европе, Центральной Азии и Сибири. Т. ІІІ. Мониторинг и моделирование ландшафтов /Под ред. В.Г.Сычева, Л. Мюллера. М., 2018: 253-257 (doi: 10.25680/3309.2018.28.99.246).

84. Полуэктов Р.А., Опарина И.В., Топаж А.Г., Миршель В. Адаптируемость динамических моделей агроэкосистем к различным почвенно-климатическим условиям. Математическое моделирование, 2000, 12(11): 3-16.

85. Dobor L., Barcza Z., Hlásny T., Árendás T., Spitky T., Fodor N. Crop planting date matters: Estimation methods and effect on future yields. Agricultural and Forest Meteorology, 2016, 223: 103-115 (doi: 10.1016/j.agrformet.2016.03.023).

86. Rötter R.P., Hoffmann M.P., Koch M., Müller C. Progress in modelling agricultural impacts of and adaptations to climate change. Current Opinion in Plant Biology, 2018, 45: 255-261 (doi: 10.1016/j.pbi.2018.05.009).

87. Bannayan M., Hoogenboom G. Weather analogue: a tool for real-time prediction of daily weather data realizations based on a modified k-nearest neighbor approach. Environmental Modelling \& Software, 2008, 23(6): 703-713 (doi: 10.1016/j.envsoft.2007.09.011).

88. Semenov M.A. Using weather generators in crop modelling. Acta Horticulturae, 2006, 707: 93 100 (doi: 10.17660/ActaHortic.2006.707.11).

89. Peleg N., Fatichi S., Paschalis A., Molnar P., Burlando P. An advanced stochastic weather generator for simulating 2-D high-resolution climate variables. Journal of Advances in Modeling Earth Systems, 2017, 9(3): 1595-1627 (doi: 10.1002/2016MS000854).

90. Maraun D., Huth R., Gutiérrez J.M., Martín D.S., Dubrovsky M., Fischer A., Widmann M. The VALUE perfect predictor experiment: evaluation of temporal variability. International Journal of Climatology, 2019, 39(9): 3786-3818 (doi: 10.1002/joc.5222).

91. Medvedev S., Topaj A., Badenko V., Terleev V. Medium-term analysis of agroecosystem sustainability under different land use practices by means of dynamic crop simulation. IFIP Advances in Information and Communication Technology, 2015, 448: 252-261 (doi: 10.1007/978-3-319-15994-2_25).

92. Dunaieva I., Mirschel W., Popovych V., Pashtetsky V., Golovastova E., Vecherkov V., Melnichuk A., Terleev V., Nikonorov A., Ginevsky R., Topaj A., Lazarev V. GIS services for agriculture monitoring and forecasting: development concept. Advances in Intelligent Systems and Computing, 2019, 983: 236-246 (doi: 10.1007/978-3-030-19868-8_24).

93. Teixeira E.I., de Ruiter J., Ausseil A.G., Daigneault A., Johnstone P., Holmes A., Tait A., Ewert F. Adapting crop rotations to climate change in regional impact modelling assessments. Science of the Total Environment, 2018, 616: $785-795$ (doi: 10.1016/j.scitotenv.2017.10.247).

94. Tayyebi A., Tayyebi A., Vaz E., Arsanjani J.J., Helbich M. Analyzing crop change scenario with the SmartScape ${ }^{\mathrm{TM}}$ spatial decision support system. Land Use Policy, 2016, 51: 41-53 (doi: 10.1016/j.landusepol.2015.11.002).

95. Palosuo T., Kersebaum K.C., Angulo C., Hlavinka P., Moriondo M., Olesen J.E., Patil R.H., Ruget F., Rumbaur C., Takáč J., Trnka M., Bindi M., Caldag B., Ewert F., Ferrise R., Mirschel W., Saylan L., Šiška B., Rötter R. Simulation of winter wheat yield and its variability in different climates of Europe: a comparison of eight crop growth models. European Journal of Agronomy, 2011, 35(3): 103-114 (doi: 10.1016/j.eja.2011.05.001).

96. Wallach D., Mearns L.O., Ruane A.C., Rötter R.P., Asseng S. Lessons from climate modeling on the design and use of ensembles for crop modeling. Climatic Change, 2016, 139(3-4): 551-564 (doi: 10.1007/s10584-016-1803-1).

97. Kollas C., Kersebaum K.C., Nendel C., Manevski K., Müller C., Palosuo T., Conradt T. Crop 
rotation modelling - a European model intercomparison. European Journal of Agronomy, 2015, 70: 98-111 (doi: 10.1016/j.eja.2015.06.007).

98. Raza A., Razzaq A., Mehmood S.S., Zou X., Zhang X., Lv Y., Xu J. Impact of climate change on crops adaptation and strategies to tackle its outcome: a review. Plants, 2019, 8(2): 34 (doi: 10.3390/plants8020034).

99. Droutsas I., Challinor A.J., Swiderski M., Semenov M.A. New modelling technique for improving crop model performance - application to the GLAM model. Environmental Modelling \& Software, 2019, 118: 187-200 (doi: 10.1016/j.envsoft.2019.05.005).

\author{
ФГБНУ Агрофизический научно-исследовательский \\ институт, \\ 195220 Россия, г. Санкт-Петербург, Гражданский просп., 14 \\ e-mail: vyakushev@agrophys.ru,mail@agrophys.com,vbadenko@gmail.com, \\ dmatveenko@inbox.ru, yuv_chesnokov@agrophys.ru $₫$
}

Поступила в редакцию

16 декабря 2019 года

Sel'skokhozyaistvennaya biologiya [Agricultural Biology], 2020, V. 55, № 3, pp. 451-467

\title{
OPERATIVE AND LONG-TERM FORECASTING OF CROP PRODUCTIVITY BASED ON MASS CALCULATIONS OF THE AGROECOSYSTEM SIMULATION MODEL IN GEOINFORMATION ENVIRONMENT
}

(review)

\section{V.P. Yakushev, V.V. Yakushev, V.L. Badenko, D.A. Matveenko, Yu.V. Chesnokov}

Agrophysical Research Institute, 14, Grazhdanskii prosp., St. Petersburg, 195220 Russia, e-mail vyakushev@agrophys.ru, mail@agrophys.com, vbadenko@gmail.com,dmatveenko@inbox.ru, yuv_chesnokov@agrophys.ru ( $\square$ corresponding author)

ORCID:

Yakushev V.P. orcid.org/0000-0002-0013-0484

Yakushev V.V. orcid.org/0000-0001-8434-5580

Badenko V.L. orcid.org/0000-0002-3054-1786

Matveenko D.A. orcid.org/0000-0002-8937-8506

The authors declare no conflict of interests

Acknowledgements:

Supported financially from the federal budget under the Agreement on subsidies of December 10, 2019 No 05.607.21.0302, the project unique identifier RFMEFI60719X0302

Received December 16, 2019

doi: 10.15389/agrobiology.2020.3.45leng

\section{Abstract}

In the context of changing socio-economic, natural and climatic conditions, there is a need for effective management tools to adapt agricultural activities. Such tools are farming systems, which are a set of interconnected agrotechnical, reclamation and organizational measures aimed at the efficient use of agrolandscapes, preservation and improvement of soil fertility, and obtaining high crop yields. The efficiency of agricultural production can be improved by using various forecasting methods based on the use of mathematical models. In crop production, statistical and dynamic simulation forecast models have been developed. The latter are more accurate and adaptive and allow you to get an answer to the question about the development of argoecosystems in the conditions of changing climatic conditions and the application of various agricultural measures. The paper provides an overview of methodological approaches for predicting crop productivity based on mass calculations of a simulation model of an agroecosystem in a geoinformation environment that can be used to justify farming systems. The analysis of the state of the problem is carried out, which presents the main current trends in the use of simulation models of agroecosystems in decision support systems for management in agriculture in general and in the support of farming systems in particular. Existing approaches and methods are classified based on spatial coverage into macro-scale, meso-scale, and micro-scale modeling methods. In the general case, these different methods require different methodological approaches are presented in the paper. The relevant basic methods and approaches for creating a universal environment for mass calculations of dynamic models of agroecosystems for different levels of spatial coverage are also presented. The analysis of the very important issue of choosing a set of control (base) points is presented where model calculations will be performed that should belong to real agricultural fields and sufficiently reflect the diversity of soil and climatic conditions of the region under consideration. Also presented are the requirements for a universal modeling environment for carrying out calculations on different models from various suppliers.

Keywords: agroecosystems, simulation modeling, mass calculations, forecasting, geographic information systems, farming systems. 